\section{Compliance, Labor-EDV}

O. Colhoun

Institut für Laboratoriumsmedizin, Klinikum Frankfurt

Höchst, Frankfurt am Main, Deutschland
Definition Im Rahmen der Labor-EDV die Erfüllung von Vorgaben, Gesetzen, Normen und Richtlinien für das medizinische Labor, z. B. Rilibäk, DIN EN ISO 15189:2007, Richtlinien des Paul-Ehrlich-Instituts für Blutprodukte oder Eurocode.

Synonym(e) Richtlinientreue 